\title{
Karolinska Institutet 200-year anniversary. Symposium on traumatic injuries in the nervous system: injuries to the spinal cord and peripheral nervous system - injuries and repair, pain problems, lesions to brachial plexus
}

\section{Mattias K. Sköld ${ }^{1,2 *}$, Mikael Svensson ${ }^{3}$, Jack Tsao $^{4,5}$, Thomas Hultgren $^{6}$, Thomas Landegren $^{6}$, Thomas Carlstedt ${ }^{6,7}$ and Staffan Cullheim ${ }^{1}$}

\author{
1 Department of Neuroscience, Karolinska Institutet, Stockholm, Sweden \\ 2 Department of Clinical Neuroscience, Department of Neurosurgery, Uppsala University Hospital, Uppsala, Sweden \\ ${ }^{3}$ Department of Clinical Neuroscience, Department Neurosurgery and Neurology, Karolinska Institutet, Karolinska University Hospital, Stockholm, Sweden \\ ${ }^{4}$ United States Navy Bureau of Medicine and Surgery, Wounded, III and Injured, Washington, DC, USA \\ ${ }^{5}$ Department of Neurology, Uniformed Services University of the Health Sciences, Bethesda, MD, USA \\ ${ }^{6}$ Section of Hand Surgery, Department of Clinical Science and Education, Karolinska Institutet, Södersjukhuset, Stockholm, Sweden \\ 7 The Peripheral Nerve Injury Unit, The Royal National Orthopaedic Hospital, University College London, London, UK
}

\section{Edited by:}

Marten Risling, Karolinska Institutet,

Sweden

\section{Reviewed by:}

Marcus Ohlsson, Karolinska Institutet, Sweden

Leif A. Havton, University of California, Los Angeles, USA

*Correspondence:

Mattias K. Sköld, Department of Neuroscience, Karolinska Institutet,

S-171 77 Stockholm, Sweden

e-mail: mattias.skold@ki.se
The Karolinska Institutet 200-year anniversary symposium on injuries to the spinal cord and peripheral nervous system gathered expertise in the spinal cord, spinal nerve, and peripheral nerve injury field spanning from molecular prerequisites for nerve regeneration to clinical methods in nerve repair and rehabilitation. The topics presented at the meeting covered findings on adult neural stem cells that when transplanted to the hypoglossal nucleus in the rat could integrate with its host and promote neuron survival. Studies on vascularization after intraspinal replantation of ventral nerve roots and microarray studies in ventral root replantation as a tool for mapping of biological patterns typical for neuronal regeneration were discussed. Different immune molecules in neurons and glia and their very specific roles in synapse plasticity after injury were presented. Novel strategies in repair of injured peripheral nerves with ethylcyanoacrylate adhesive showed functional recovery comparable to that of conventional epineural sutures. Various aspects on surgical techniques which are available to improve function of the limb, once the nerve regeneration after brachial plexus lesions and repair has reached its limit were presented. Moreover, neurogenic pain after amputation and its treatment with mirror therapy were shown to be followed by dramatic decrease in phantom limb pain. Finally clinical experiences on surgical techniques to repair avulsed spinal nerve root and the motoric as well as sensoric regain of function were presented.

Keywords: spinal cord, peripheral nerves, nerve injury, mirror therapy, neural stem cell

\section{ADULT NEURAL STEM/PROGENITOR CELLS TRANSPLANTED TO THE HYPOGLOSSAL NUCLEUS OF RAT INTEGRATES WITH THE HOST CNS AND PROMOTES MOTOR NEURON SURVIVAL Michael Fagerlund, Cynthia Perez Estrada, Nasren Jaff, Lou Brundin and Mikael Svensson}

Transplantation of neural stem cells and the mobilization of endogenous neuronal precursors in the adult brain have been proposed as therapeutic strategies for a large range of central nervous system disorders and injuries. The aim of the present study was to investigate the possible survival and integration of grafted neural progenitor cells (NPCs) from the subventricular zone (SVZ) in a hypoglossal nerve avulsion model with substantial neuronal loss.

Adult NPCs from the SVZ were cultured from inbred transgenic eGFP Lewis rats and transplanted to the hypoglossal nucleus of inbred Lewis rat from the same family but that were not carrying the eGFP strain after avulsion of the hypoglossal nerve. Grafted cells survived in the host more than 3 months and differentiated into neurons ( $\beta$-III tubulin) with fine axon- and dendrite-like processess as well as astrocytes (GFAP) and oligodendrocytes (O4) with typical morphology. Stainings for synaptic structures (synaptophysin and bassoon) indicated integration of differentiated cells from the graft with the host CNS. Furthermore, transplantation of NPCs increased the number of surviving motor neurons in the hypoglossal nucleus after nerve avulsion that, if untreated, result in substantial neuronal death. The NPCs used in this study expressed VEGF in vitro as well as in vivo following transplantation that may promote neuronal survival.

\section{PLEASE MIND THE GAP - AN UPDATE ON EXPERIMENTAL} CNS-PNS BRIDGING EFFORTS IN VENTRAL SPINAL CORD Mattias K. Sköld, T. Ochsman, T. Carlstedt, H. Lindå, S. Plantman, E. Rostami, M. Angeria and M. Risling Replantation of avulsed spinal ventral roots has been shown to enable significant and useful regrowth of motor axons in both experimental animals and in human clinical cases, making up an interesting exception to the rule of unsuccessful neuronal regeneration in CNS. 
The CNS/PNS transitional zone is a region normally relatively scarce of blood vessels, with few capillaries crossing the CNS/PNS border, but it has been shown that lesions to this area is followed by upregulation of vascular growth factors and angiogenesis (Skold et al., 2000). Increasing interest during recent years has focused on the fact that blood vessels and nerves share common growth factors and growth patterns during development and in disease (Carmeliet and Tessier-Lavigne, 2005). Therefore we investigated the vascular supply in the area of ventral root avulsion and replantation. The left L5 ventral root was identified and avulsed by gentle traction of the root followed by replantation of the avulsed root into the lateral funiculus of the spinal cord. We found an increased number of capillaries in the CNS/PNS transitional zone after replantation and in addition that these capillaries overgrow the CNS/PNS border but also that astrocytes in this scar area are organized alongside the blood vessels, typically in a cone shaped fashion resembeling of the normal morpholgy in the trasitional zone. At the replantation site and in the more central parts of the spinal cord just adjacent to the replantation site, axons and astrocytes were seen growing alongside each other, a pattern that could also be observed in more peripheral parts of the replanted ventral root where axons and astrocytic processess do align (Figure 1A). Furthermore, we can show that neurites do regrow from the spinal cord ventral horn, through the scar tissue and out into the ventral roots and that these regrowing axons are organized alongside blood vessels growing from the central parts of the spinal cord bridging the border between CNS and PNS and growing in to the replanted ventral root (Figure 1B). In conclusion it seems that the normally rather avascular zone between the CNS-PNS at the transitional zone is vascularized after replantation of avulsed ventral roots and that the vessels, together with astrocytes and neurites do grow from CNS to PNS. Further experiments are needed to analyze the possible interplay between these different cell types in this kind of injury.
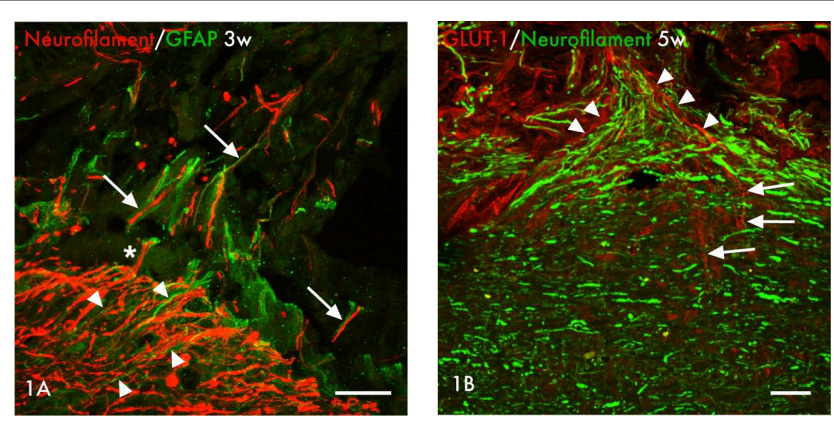

FIGURE 1 | (A) Axons (neurofilament, red) and astrocytes (GFAP, green) are seen at the border between the central and peripheral parts of the spinal cord at the site of replantation of the avulsed ventral root at 3 weeks after injury. In the more central parts astrocyte processes can be seen growing alongside axons (arrowheads) in a pattern that can also be found in the more peripheral parts where axons and astrocytic processess do align (arrows). Marked is also one single axon that seemingly grow from the central parts of the cord in to the peripheral nerve graft alongside astrocytic processess (asterisk*). Scale bar $50 \mu \mathrm{m}$. (B) Micrographs showing the relation between regenerating blood vessels, shown with marker for GLUT-1 (red), and regenerating axons, shown with marker for neurofilament (green), at the replantation site at 5 weeks after replantation of avulsed ventral roots. Note how blood vessels grow from the CNS compartment (arrows) to the PNS compartment (arrowheads) alongside the axons (green). Scale bar $50 \mu \mathrm{m}$.
We did also analyze the acute response to ventral root avulsion and replantation in the ventral quadrant of spinal cord tissue at the injured side from adult rats with gene arrays in combination with cluster analysis of gene ontology search terms and can show significant differences between rats subjected to ventral replantation compared to avulsion only. The number of genes related to cell death is similar in the two models after $24 \mathrm{~h}$, but a significantly larger number of genes related to neurite growth and development are regulated in rats treated with ventral root replantation, possibly reflecting intrinsic neuroregenerative capacity in the replantation model. In addition, regulation of genes related to synaptic transmission was much more pronounced after replantation than after avulsion without replantation. These data indicate that the axonal regenerative response from replantation is initiated at an earlier stage than differences in terms of neuron survival (Risling et al., 2011). An analysis such as this could possibly facilitate the comparison of the regenerative response in different kind of neurotraumatic injuries.

\section{IMMUNE MOLECULES IN NEURONS AND GLIA - IMPLICATIONS FOR SYNAPTIC PLASTICITY AFTER NERVE LESION}

\section{Staffan Cullheim}

There is accumulating evidence that immune molecules are used by neurons and glia in the CNS to modulate synaptic function and plasticity during development and after nerve injury. Spinal motoneurons in rat and mouse constitutively express mRNAs for the major histocompatibility complex (MHC) class I and beta2microglobulin (beta2m), which is necessary for surface expression of MHC class I. One classical response to lesion of motor axons is the shedding of synapses from the cell surface of severed motoneurons. We have shown that mice lacking the beta $2 \mathrm{~m}$ gene display a more extensive synaptic elimination from the cell bodies of axotomized spinal motoneurons compared with wild-type (WT) mice. This surplus elimination is directed toward synaptic terminals with a putative inhibitory function (Oliveira et al., 2004).

The complement system is a part of the innate immune system. Mice lacking complement proteins $\mathrm{C} 1 \mathrm{q}$ or $\mathrm{C} 3$ exhibit large defects in the organization of synapses in the development of the retinogeniculate pathway, resembling the picture seen in the absence of MHC class I (Stevens et al., 2007). We therefore studied the synaptic elimination from axotomized motoneurons in mice deficient in C3. In these mice the elimination was much less prominent than in WT animals, which lends support to the idea that complement proteins are "tagging" synapses to be eliminated.

Astrocytes and, in particular, microglia have been attributed important roles in the synaptic elimination response after axon lesion. In mice, mRNAs for classical MHC class I $(\mathrm{H} 2-\mathrm{Kb} / \mathrm{Db})$ was upregulated in both motoneurons and microglia after lesion, while non-classical MHC class I (H2-T22) was upregulated only in motoneurons. In contrast, C1q mRNA was upregulated solely in activated microglia, with no neuronal expression. The general pattern of activation, as revealed by GFAP and Iba 1 immunoreactivity for astrocytes and microglia, respectively, did not reveal any major differences between WT animals and mice with gene deletions for MHC class I or complement proteins. We conclude that the effects exerted by the immune molecules are very specific, probably involves glia but are not mirrored by the general activation pattern of glia. 
LONG-TERM RESULTS OF PERIPHERAL NERVE REPAIR: A COMPARISON OF NERVE ANASTOMOSIS WITH ETHYLCYANOACRYLATE AND EPINEURAL SUTURES Thomas Landegren, Anders Sondén, Mårten Risling and Jonas K. Persson

There is a need for complementary surgical techniques which offers rapid and reliable primary repair of transected nerves. Anastomosis of a nerve with synthetic adhesive following a lesion has previously been shown to indicate recovery to an extent comparable to that of conventional techniques. The aim of this study was to quantify the morphological and functional recovery and evaluate the selectivity of muscle reinnervation after transection and repair of rat sciatic nerve, and compare epineural sutures with a synthetic ethyl-cyanoacrylate tissue adhesive.

Six months after repair, when reinnervation had well been completed the tibial branch to the lateral gastrocnemius muscle and the caudal sural cutaneous nerve were examined with electrophysiological measurements of motor and sensory conduction velocity, motor nerve action potentials, and quantitative histological examinations. Furthermore, cholera toxin B technique of retrograde axonal tracing was used to evaluate the morphology, the number, and the three-dimensional location of a-motoneurons in L5 spinal cord, innervating the lateral gastrocnemius muscle and the results were put in relation to the recorded wet weight of the muscle.

There was functional reinnervation of motor and sensory nerves in both groups, as shown by equivalent recovery of motor and sensory conduction velocities, and motor nerve action potentials. Histological examination showed no significant difference in the mean diameter, fiber density, or the number of regenerated myelinated motor and sensory axons distal to the repair site between the two groups. Moreover, independent of repair method, the redistribution of the motoneuron pool were markedly disorganized, had increased apparently in number, and were scattered throughout a larger volume of the spinal cord gray matter with a decrease in the synaptic coverage compared to controls. A reduction in muscle weight was observed as well. Differences compared to controls were statistically significant.

We conclude that anastomosis of the nerve with ethylcyanoacrylate adhesive supports morphological and functional recovery comparable to that of conventional epineural sutures after a unilateral lesion of the sciatic nerve in adult rats.

\section{SURGICAL TREATMENT OF RESIDUAL IMPAIRMENT AFTER BRACHIAL PLEXUS LESIONS \\ Tomas Hultgren}

The basic strategies and techniques for surgical repair of brachial plexus lesions using multiple nerve grafts were essentially established in the 1980s. Additional techniques such as nerve transfer ("neurotization"), and spinal root reimplantation as pioneered by Prof. Carlstedt, have led to improved results. Even so, these often very extensive nerve injuries can be expected to leave major functional loss. This presentation will outline some of the surgical techniques which are available to improve function of the limb, once the nerve regeneration has reached its limit.

The functional impairment following obstetric plexus (OBP) lesions differs somewhat from the adult injuries. Contractures are common, and hypoplasia, dysplasia, and joint subluxations are often seen in the growing child as sequels to an OBP lesion. The shoulder joint is particularly susceptible to such deformities. Contractures and deformities of the shoulder in OBP patients were described already 100 years ago, and yet there is no consensus regarding treatment. I will present results from our ongoing prospective studies which aim to provide a scientific basis for optimal surgical treatment of these patients.

\section{USE OF MIRROR THERAPY FOR THE TREATMENT OF PHANTOM LIMB PAIN \\ JackW.Tsao, Ilana R. Yurkiewicz, Brett Monson, Brenda L. Chan, Lindsay Hussey-Andersen, Katie E. Hughes, Richard Witt, Kenneth M. Heilman and Paul F. Pasquina}

\section{INTRODUCTION}

At least $90 \%$ of amputees experience phantom limb pain (PLP; Melzack, 1990). This pain can manifest as a variety of sensations, including burning, stabbing, and squeezing (Jensen et al., 1985). One proposed explanation for PLP is the presence of a conflict between visual and proprioceptive inputs to the brain. Mirror therapy intends to reduce this conflict by having an amputee perform specific movements with the phantom limb while viewing the reflected image of the intact limb executing actual movements (Ramachandran and Hirstein, 1998). While this therapy was shown to treat chronic phantom limb pain in $60 \%$ of upper limb amputees (Ramachandran and Rogers-Ramachandran, 1996), studies with lower limb amputees were limited. Moreover, it remains unclear whether the therapy's efficacy varies in response to the diverse manifestations of PLP. We performed a randomized, sham-controlled study to explore the efficacy of short-term use of mirror therapy for reducing PLP in lower extremity amputees (Chan et al., 2007). This paper expands upon our findings and examines which types of PLP are most responsive to mirror therapy.

\section{MATERIALS AND METHODS}

We randomly assigned 22 unilateral lower limb amputees with PLP to three treatment groups. Mirror group subjects performed movements with the phantom limb while viewing the reflected image of their intact limb moving (Figure 2). Covered mirror group subjects performed the exercise with the mirror covered by an opaque sheet. Mental visualization group subjects had no mirror, but simply imagined performing the movements with the phantom limb. All subjects engaged in the exercises for $15 \mathrm{~min}$ daily. Each day for 4 weeks, subjects monitored the duration and intensity of pain episodes using a $100-\mathrm{mm}$ visual analog scale (VAS) and reported their specific manifestation of pain with the McGill Pain Assessment Questionnaire Short-Form. After the first month, subjects in the covered mirror and mental visualization groups were crossed over to mirror therapy.

\section{RESULTS}

Eighteen subjects completed the study. All six subjects (100\%) in the mirror group experienced a reduction in PLP, in contrast with one patient $(17 \%)$ in the covered mirror group and two patients (33\%) in the mental visualization group. After crossing over to mirror therapy, PLP decreased in in 8 of 9 subjects (89\%) at 2 months, yielding a total of 14 of 15 subjects (93.3\%) who used the mirror ultimately reported 


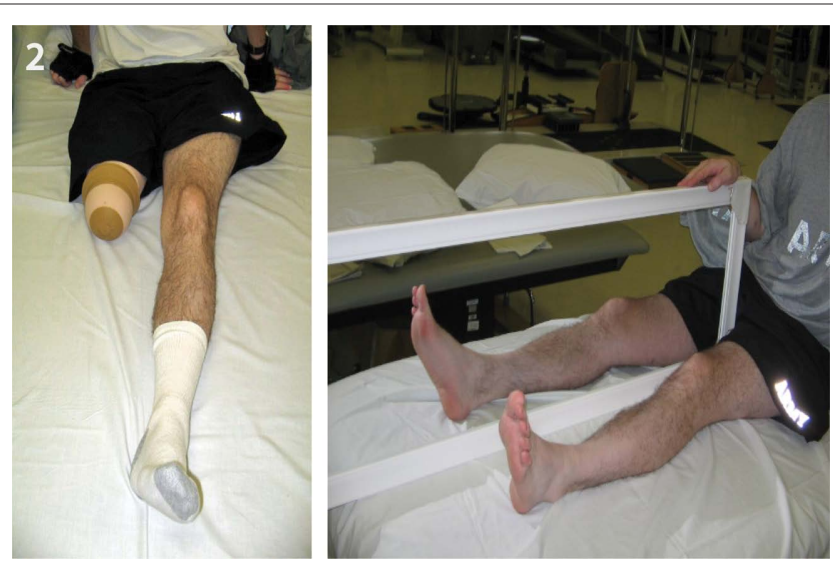

FIGURE 2 | Right above-knee amputee using the mirror.

decreased PLP (Figure 3; Chan et al., 2007). We found a significant effect of the number of mirror treatment days $(p<0.0001)$, with efficacy seen starting at 5 days $(p=0.023)$. A treatment effect was seen for four types of pain: shooting $(p=0.007)$, stabbing $(p=0.003)$, sharp $(p<0.0001)$, and aching $(p<0.0001$; Figure 4). Throbbing,

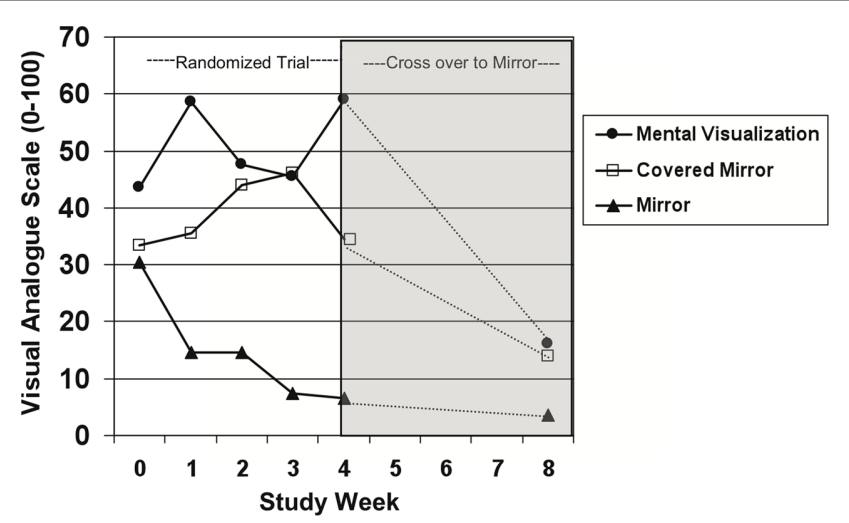

FIGURE 3 | Change in pain in subjects measured using the VAS. Group medians are depicted for each time point. After the first month (week 4), subjects in the covered mirror and mental visualization groups were switched to using the uncovered mirror. $N=6$ per group after accounting for dropouts. Reprinted with permission from the New England Journal of Medicine (Chan et al., 2007).

cramping, gnawing, hot-burning, heavy, tender, and splitting pain also decreased, but these did so independently from the number of treatment days. In addition, length of PLP episodes decreased
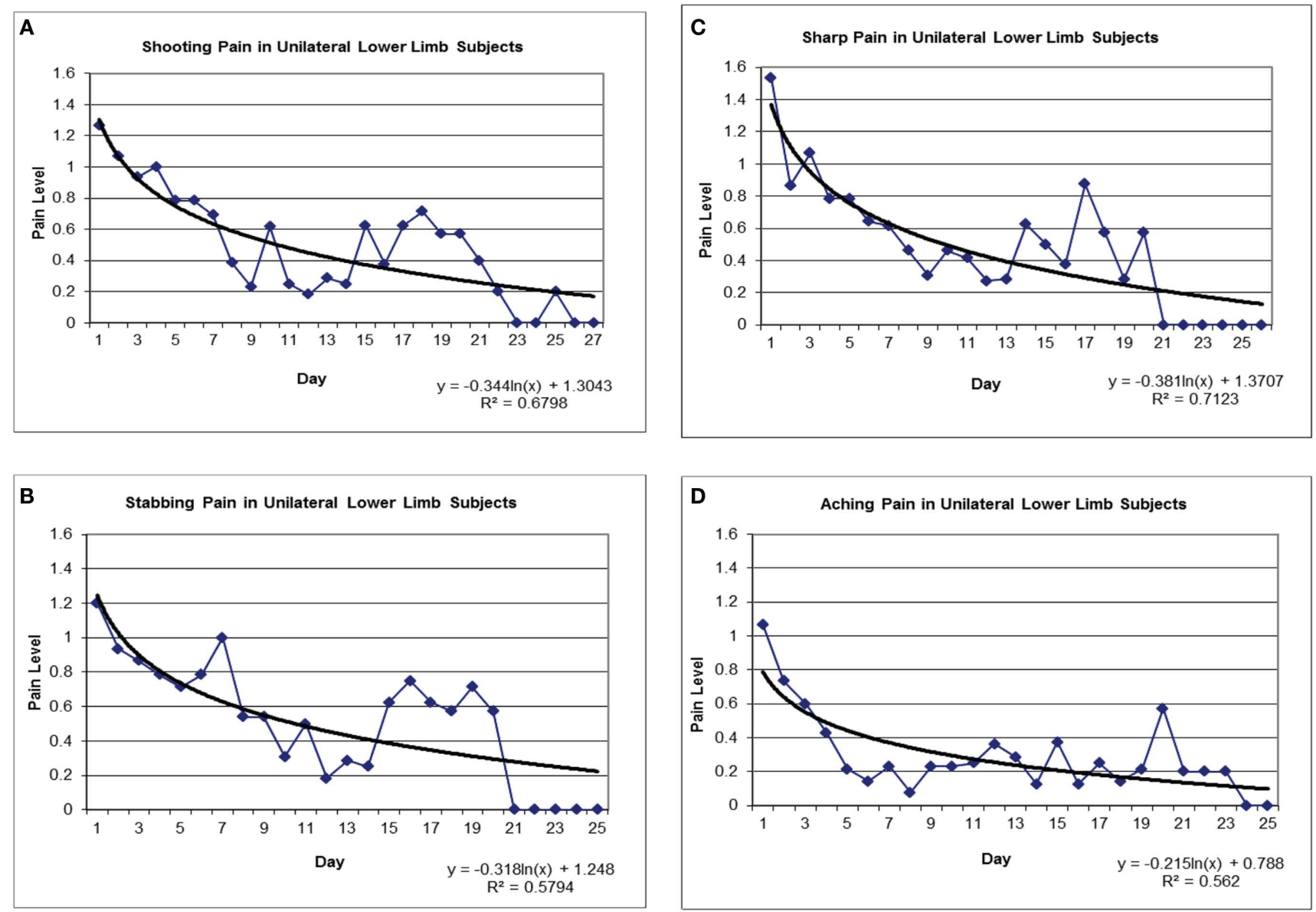

FIGURE 4 | Treatment effect for shooting pain (A), stabbing pain (B), sharp pain (C), and aching pain (D). 
after the first week while the number of daily PLP episodes did not begin to decrease until the third week (data not shown). Of those who reported improvement in pain, pain levels at 4 months after starting therapy were minimal or non-existent, and several subjects seen in routine clinical follow-up 2 years later remained pain-free.

\section{CONCLUSION}

These results suggest that visual input is necessary for mirror therapy to be effective, providing additional support for a causative role for mismatch between visual and proprioceptive signals in the brain in the etiology of PLP. Our findings are also the first to offer insight into the efficaciousness of mirror therapy broken down by specific phantom pain type. We hope this information may be valuable for tailoring mirror therapy to those who will benefit most.

\section{SPINAL CORD SURGERY AFTER ROOT AVULSION INJURY IN MAN RESULTS IN MOTOR AND SENSORY RECOVERY Thomas Carlstedt}

Spinal nerve root avulsion is a longitudinal spinal cord injury that affects mainly the final common motor pathway and the primary sensory medullary trajectories. In humans this occurs most frequently in traction injuries to the brachial plexus but also in trauma to the lumbosacral plexus and cauda equina as well as conus medullaris. This injury has serious effects on the pertinent spinal cord segment with the breakdown of connections and networks, death of motor, autonomic and sensory neurons, and the development of a spinal cord scar. This injury often occurs as a result of road traffic accidents or violent acts. About 1000 patients are affected annually in the UK. The functional consequence of such injury is lower motoneuron syndromes, associated with autonomic paralysis including dysfunctional internal organs, limb muscle atrophy, sensory impairment, and chronic pain. Spinal nerve root injury has been associated with an overall poor clinical outcome as a successful surgical repair would require axonal regrowth within the central nervous system as spinal cord regeneration. A surgical technique

\section{REFERENCES}

Carlstedt, T., Hultgren, T., Nyman, T., and Hansson, T. (2009). Cortical activity and hand function restoration in a patient after spinal cord surgery. Nat. Rev. Neurol. 5, 571-574.

Carmeliet, P., and Tessier-Lavigne, M. (2005). Common mechanisms of nerve and blood vessel wiring. Nature 436, 193-200.

Chan, B. L., Witt, R., Charrow, A. P., Magee, A., Howard, R., Pasquina, P. F., Heilman, K. M., and Tsao, J. W. (2007). Mirror therapy for phantom limb pain. N. Engl. J. Med. 357, 2206-2207.

Havton, L. A., and Carlstedt, T.(2009). Repair and rehabilitation of plexus and root avulsions in animal models and patients. Curr. Opin. Neurol. 22, 570-574.

Jensen, T. S., Krebs, B., Nielsen, J., and Rasmussen, P. (1985). Immediate and long-term phantom limb pain in amputees: incidence, clinical charac- teristics and relationship to pre-amputation limb pain. Pain 21, 267-278.

Melzack, R.(1990). Phantom limbs and the concept of a neuromatrix. Trends Neurosci. 13, 88-92.

Oliveira, A. L., Thams, S., Lidman, O., Piehl, F., Hokfelt, T., Karre, K., Linda, H., and Cullheim, S. (2004). A role for MHC class I molecules in synaptic plasticity and regeneration of neurons after axotomy. Proc. Natl. Acad. Sci. USA, 101, 17843-17848.

Ramachandran, V. S., and Hirstein, W.(1998). The perception of phantom limbs. The D. O. Hebb lecture. Brain 121, 1603-1630.

Ramachandran, V. S., and RogersRamachandran, D. (1996). Synaesthesia in phantom limbs induced with mirrors. Proc. Biol. Sci. 263, 377-386.

Risling, M., Ochsman, T., Carlstedt, T., Linda, H., Plantman, S., Rostami, E., Angeria, M., and Skold, M. K. (2011). On acute gene expression changes after

for the repair of this spinal cord injury was developed from basic science experiments and successfully translated to humans (Havton and Carlstedt, 2009).

Today, the reimplantation of avulsed ventral roots to the spinal cord in total brachial plexus avulsion injury restores useful proximal limb function and is the method of choice in the treatment of such devastating injuries. Even hand function can be restored by this technique. By means of functional magnetic resonance imaging (fMRI) it was established that the restored hand function relayed on pre-injury established cortical sensory program (Carlstedt et al., 2009).

Sensory roots are not possible to replant with functional recovery. Sensory recovery is therefore not occurring. In some cases as an expression of plasticity there are phenomenona observed as referrals of sensation to the denervated limb as well as hypersensitivity at border zones between normal and denervated dermatomes. When nerve graft has been implanted into the dorsal part of the spinal cord it has been possible to record return of some aspects of sensation. There was in such a case possible to elicit a biceps reflex and also record by means of electrophysiology a H-reflex proving that by this surgical technique it has been possible to restore a sensory-motor spinal cord reflex. However, neither quantitative sensory testing (QST), nor contact heat evoked potential stimulation (CHEPs) could demonstrate any exteroceptive sensory qualities.

Pain, which is most severe and excruciating in most cases of brachial plexus avulsion injuries, was found to be alleviated in conjunction with motor recovery after motor (but not sensory) root replantation. The mechanisms behind this are elusive but recent observations have indicated that there is retrograde transneuronal degeneration into the spinal cord dorsal horn following ventral root avulsion. This is reversed by ventral root replantation.

These observations achieved by spinal cord surgery will serve as baseline data when assessing the effects of future application of adjuvant therapies.

ventral root replantation. Front. Neur. 1: 159. doi: 10.3389/fneur.2010.00159 Skold, M., Cullheim, S., Hammarberg, H., Piehl, F., Suneson, A., Lake, S., Sjogren, A., Walum, E., and Risling, M. (2000). Induction of VEGF and VEGF receptors in the spinal cord after mechanical spinal injury and prostaglandin administration. Eur. J. Neurosci. 12, 3675-3686.

Stevens, B., Allen, N. J., Vazquez, L. E., Howell, G. R., Christopherson, K. S., Nouri, N., Micheva, K. D., Mehalow, A. K., Huberman, A. D., Stafford, B., Sher, A., Litke, A. M., Lambris, J. D., Smith, S. J., John, S. W., and Barres, B. A. (2007). The classical complement cascade mediates CNS synapse elimination. Cell 131, 1164-1178.

Conflict of Interest Statement: The authors declare that the research was conducted in the absence of any commercial or financial relationships that could be construed as a potential conflict of interest.
Received: 02 February 2011; paper pending published: 23 February 2011; accepted: 25 April 2011; published online: 12 May 2011.

Citation: Sköld MK, Svensson M, Tsao J, Hultgren T, Landegren T, Carlstedt T and Cullheim S (2011) Karolinska Institutet 200-year anniversary. Symposium on traumatic injuries in the nervous system: injuries to the spinal cord and peripheral nervous system - injuries and repair, pain problems, lesions to brachial plexus. Front. Neur. 2:29. doi: 10.3389/fneur.2011.00029 This article was submitted to Frontiers in Neurotrauma, a specialty of Frontiers in Neurology.

Copyright (C) 2011 Sköld, Svensson, Tsao, Hultgren, Landegren, Carlstedt and Cullheim. This is an open-access article subject to a non-exclusive license between the authors and Frontiers Media SA, which permits use, distribution and reproduction in other forums, provided the original authors and source are credited and other Frontiers conditions are complied with. 